\title{
Erratum to: Altered NR4A Subfamily Gene Expression Level in Peripheral Blood of Parkinson's and Alzheimer's Disease Patients
}

\author{
Francesca Montarolo $^{1}$ - Simona Perga ${ }^{1}$ - Serena Martire ${ }^{1}$ - De'sire'e Nicole Navone ${ }^{1}$. \\ Alberto Marchet ${ }^{2}$ - Daniela Leotta ${ }^{2}$ - Antonio Bertolotto ${ }^{1}$
}

Published online: 2 December 2016

(C) Springer Science+Business Media New York 2016

Erratum to: Neurotox Res (2016) 30:338-344

DOI 10.1007/s12640-016-9626-4

The original version of this article unfortunately contained a mistake. Some of the values in Table 2 are not correctly presented. The corrected version of Table 2 is given below.

Table 2 Clinical characteristics of enrolled $\mathrm{PD}$ and $\mathrm{AD}$ populations

\begin{tabular}{|c|c|c|c|c|c|c|}
\hline & \multicolumn{2}{|l|}{ PD } & \multicolumn{4}{|l|}{$\mathrm{AD}$} \\
\hline $\mathrm{N}$ & \multicolumn{2}{|l|}{20} & \multicolumn{4}{|l|}{22} \\
\hline Disease onset (y) median (range) & \multicolumn{2}{|c|}{$61.5(36-75)$} & \multicolumn{4}{|c|}{$65.0(52-81)$} \\
\hline \multirow[t]{2}{*}{ Familiarity N (\%) } & yes & $4(20)$ & $3(9.2)$ & & & \\
\hline & no & $16(80)$ & $19(81.8)$ & & & \\
\hline Patients treated with specific therapies & L-DOPA & L-DOPA + Dopamine agonists & Donepezil & Memantine & Rivastigmine & $\begin{array}{l}\text { Combination of } \\
\text { two of them }\end{array}$ \\
\hline $\mathrm{N}(\%)$ & $19(95)$ & $16(80)$ & $11(50)$ & $6(27.3)$ & $4(18.2)$ & $4(18.2)$ \\
\hline UPDRS at least part III median (range) & \multicolumn{2}{|c|}{$33.5(78-16)$} & \multicolumn{4}{|l|}{-} \\
\hline H\&Y scale median (range) & \multicolumn{2}{|c|}{$2.7(4-1.5)$} & - & & & \\
\hline
\end{tabular}

$H C$ healthy controls, $P D$ Parkinson's disease, $A D$, Alzheimer's disease, $y$ years, $N$ number of subjects, UPDRS unified Parkinson's disease rating scale, $H \& Y$ Hoehn and Yahr scale

The online version of the original article can be found at http://dx.doi. org/10.1007/s12640-016-9626-4

Francesca Montarolo

francesca.montarolo@unito.it

1 Neurobiology Unit, Neurologia 2 - CReSM (Regional Referring

Center of Multiple Sclerosis), Neuroscience Institute Cavalieri

Ottolenghi (NICO), University of Turin \& AOU San Luigi Gonzaga,

Regione Gonzole 10, 10043 Orbassano, TO, Italy

2 Division of Neurology, Martini Hospital, Via Tofane 71,

10141 Turin, TO, Italy 